\title{
Update on first-line treatment of advanced ovarian carcinoma
}

This article was published in the following Dove Press journal:

International Journal of Women's Health

24 January 2013

Number of times this article has been viewed

Z Kemp'

JA Ledermann ${ }^{1,2}$

'Department of Oncology, University College London Hospitals, ${ }^{2}$ University College London Cancer Institute, University College London, London, United Kingdom
Correspondence: Jonathan A Ledermann Cancer Research UK and UCL Cancer Trials Centre, University College London, 90 Tottenham Court Road, London,

WIT 4TJ, United Kingdom

Tel +44020 76799898

Fax +4402076799899

Email j.ledermann@ucl.ac.uk
Abstract: Despite the high response rate to first-line treatment of advanced ovarian cancer, the vast majority of patients relapse. Maximal debulking surgery and chemotherapy with a platinum doublet have remained the standard of care for many years and new approaches are imperative. Recent clinical trials have given grounds for hope. Neoadjuvant chemotherapy, intraperitoneal delivery, and dose-dense strategies have all shown promising results, as has the targeting of angiogenesis. A greater understanding of the molecular landscape of ovarian cancer is helping to identify new treatment options. In this review, we will highlight the key trials and recent progress in these areas.

Keywords: ovarian cancer, first-line therapy, neoadjuvant, intraperitoneal, dose-dense, angiogenesis

\section{Introduction}

The survival figures for ovarian cancer are the poorest amongst the gynecological cancers. In Europe, there are an estimated 65,697 new cases and 41,448 deaths each year. ${ }^{1}$ A minority of women (approximately $15 \%$ ) present with disease confined to the ovaries and following surgery their 5 -year survival is more than $90 \%$. For those presenting with advanced disease (FIGO stage III-IV), the outlook is less optimistic with the probability of surviving 5 years being less than $30 \% .^{2}$ Early diagnosis of ovarian cancer is therefore highly desirable but confounded by the lack of clearly defined symptoms. ${ }^{3,4}$ Trials have been undertaken to assess the efficacy of screening asymptomatic women with annual CA 125 and transvaginal ultrasound but, to date, these have failed to demonstrate a reduction in mortality. ${ }^{5}$ The ongoing UK Collaborative Trial of Ovarian Cancer Screening will report in the next 2 years. ${ }^{6}$

For advanced disease, the standard treatment of surgery and platinum-based chemotherapy has remained unchanged for many years. Attempts to improve the platinum doublet by addition of a third cytotoxic agent or very high-dose therapy have only increased toxicity without benefit in progression-free or overall survival.

In this review, we will consider how new approaches to the standard of care of carboplatin and paclitaxel, such as neoadjuvant chemotherapy, intraperitoneal treatment, dose-dense therapy, and targeting angiogenesis, are impacting on the outcome of ovarian cancer. However, it may be that as our understanding of the heterogeneous nature of ovarian cancer deepens, we will also need to focus our attention on the genomic landscape of the disease if we are to improve treatment. 


\section{Standard chemotherapy}

Since the 1970s, the standard of care for the management of advanced ovarian cancer has been maximum cytoreductive surgery and platinum-based chemotherapy, with carboplatin combinations replacing cisplatin-based chemotherapy over the last 15 years.

Although platinum drugs are the most active agents in ovarian cancer, taxanes have emerged as an important group of drugs, particularly when given in conjunction with platinum. This was first demonstrated in 1996 following publication of the Gynecologic Oncology Group (GOG) 111 trial which reported the superiority of cisplatin and paclitaxel compared with cisplatin and cyclophosphamide. ${ }^{7}$ Mature data from this and a similar study, the European-Canadian Intergroup trial (OV10), ${ }^{8}$ showed in each case an $11 \%$ absolute gain favoring the paclitaxel combination. Carboplatin with paclitaxel was subsequently found to be at least as effective as cisplatin and paclitaxel. ${ }^{9}$ The use of docetaxel as a substitute for paclitaxel gives similar progression-free and overall survival data and less neurotoxicity, but at the risk of dose-limiting myelosuppression. ${ }^{10}$

However, two studies have questioned the additional value of paclitaxel. The International Collaborative Ovarian Neoplasm (ICON-3) study compared carboplatin and paclitaxel with single-agent carboplatin or cyclophosphamide, doxorubicin, and cisplatin, ${ }^{11}$ and did not show any survival advantage. Some have attributed this to the inclusion of a greater number of low-risk patients than GOG 111 and OV10, which included a larger percentage of stage III and IV suboptimally debulked patients. A second trial, GOG 132, included a comparison of cisplatin with cisplatin and paclitaxel. Patients receiving cisplatin crossed over to paclitaxel on progression. There was no difference in overall survival between these two arms. ${ }^{12}$ Nevertheless, carboplatin and paclitaxel have become the established standard of care and the favored comparator arm in clinical trials. ${ }^{13}$

Although the response rate for first-line carboplatin and paclitaxel is $70 \%-80 \%$, with more than $50 \%$ achieving complete remission after surgery and chemotherapy, the majority of women with advanced ovarian cancer will subsequently relapse or progress and eventually develop chemotherapyresistant disease. A number of randomized trials have sought to improve survival by addition of a third agent. The largest of these studies was GOG 182/ICON-5, an international collaboration which sought to evaluate three drugs with well defined activity in the setting of recurrent ovarian cancer, ie, topotecan, gemcitabine, and pegylated liposomal doxorubicin. ${ }^{14}$ However, at the planned interim analysis in 2004, none of the experimental arms had met the prespecified reduction in progression-free survival compared with the reference arm, and the study was closed.

\section{Neoadjuvant chemotherapy}

Patients who undergo maximal cytoreductive surgery with 0-1 $\mathrm{cm}$ residual tumor have the best survival. In some patients, optimal cytoreduction is difficult and a second (interval) debulking surgery is performed. This has been shown to improve overall survival. ${ }^{15}$ This randomized trial is a key publication demonstrating the value of cytoreductive surgery. In order to avoid two operations, an approach of primary (neoadjuvant) chemotherapy has been explored to reduce the tumor burden and facilitate definitive surgery, which takes place after 3-4 cycles of chemotherapy. This has the potential to minimize the invasiveness of surgery without reducing its efficacy, a strategy used to facilitate breast-conserving surgery in patients with breast cancer.

The strategy of neoadjuvant chemotherapy has been tested in a randomized trial, ie, the European Organisation for Research and Treatment of Cancer (EORTC) 55971, in potentially operable patients with stage IIIc or IV ovarian, fallopian tube, or primary peritoneal carcinoma. They were randomized between standard primary debulking surgery and chemotherapy or neoadjuvant chemotherapy with interval debulking surgery. ${ }^{16}$ Following surgery, residual tumor $\leq$ $1 \mathrm{~cm}$ was achieved in $80.6 \%$ of those receiving neoadjuvant chemotherapy but only $41.6 \%$ of those receiving standard upfront surgery, who also suffered more postoperative adverse events and mortality. Progression-free and overall survival were similar in the two arms, but the strongest independent variable for predicting overall survival was complete resection of all macroscopic disease.

Two further prospective trials have already completed accrual and results will soon be reported. The CHORUS (CHemotherapy OR Up front Surgery) trial in the UK has a design similar to that of EORTC 55971, and the results from both studies will be pooled to provide a large randomized dataset. In the Japanese study, JCOG 0602, the trial design consists of a total of eight cycles of chemotherapy, with either eight given after primary surgery or four given neoadjuvantly before interval debulking and four afterwards..$^{17,18}$

The conclusion drawn from EORTC 55971 is that delaying surgery by giving primary chemotherapy does not impact negatively on survival. The absence of residual disease after surgery remains important, and primary surgery is still advocated unless it is considered by preoperative assessment that it would be difficult to "debulk" the tumor to no 
macroscopic residual disease, either due to the location or extent of disease, or because the resultant morbidity would be considered too great. Many published series advocate that with an "aggressive" approach, complete "debulking" is possible in most cases. However, these reports may be influenced by selection bias; amongst the centers participating in the EORTC 55971 study, there was considerable variation in the completeness of primary cytoreduction. It is likely that primary chemotherapy will become particularly important for those with bulky stage IIIc disease, particularly involving the upper abdomen, or stage IV disease, ${ }^{18,19}$ and it is estimated that in routine clinical practice about one third of patients undergo neoadjuvant treatment. Therefore, it is essential that this approach becomes incorporated into the design of clinical trials investigating new therapies.

\section{Intraperitoneal chemotherapy}

Advanced ovarian cancer is predominantly a disease of the peritoneal surfaces, and this has prompted investigation into delivery of chemotherapy directly into the peritoneum. This increases the intensity of the dose delivered to the tumor without compromising plasma drug levels and potentially spares normal tissues such as bone marrow from increased toxicity.

Evidence to support the use of intraperitoneal chemotherapy is derived from three randomized trials. The GOG 104 study examined intravenous cyclophosphamide $600 \mathrm{mg} / \mathrm{m}^{2}$ with either intravenous or intraperitoneal cisplatin $100 \mathrm{mg} / \mathrm{m}^{2}$ administered every 3 weeks for six cycles. ${ }^{17}$ The intraperitoneal group showed an estimated median survival of 49 months compared with 41 months in the intravenous group but experienced more frequent moderate to severe abdominal pain.

In GOG 114, patients received either six cycles of intravenous paclitaxel $135 \mathrm{mg} / \mathrm{m}^{2}$ and cisplatin $75 \mathrm{mg} / \mathrm{m}^{2}$ every 3 weeks or intravenous carboplatin (AUC 9) every 28 days for two cycles, followed by six 3-weekly cycles of intravenous paclitaxel $135 \mathrm{mg} / \mathrm{m}^{2}$ and intraperitoneal cisplatin $100 \mathrm{mg} / \mathrm{m}^{2} .{ }^{20}$ The intraperitoneal arm demonstrated improved progression-free survival (median 28 versus 22 months; hazards ratio $[\mathrm{HR}] 0.78 ; P=0.01$ ) and overall survival (median 63 versus 52 months; HR $0.81 ; P=0.05$ ) but increased toxicity, with $18 \%$ of patients receiving fewer than two courses of intraperitoneal chemotherapy.

The most impressive median survival data following intraperitoneal chemotherapy comes from the GOG 172 study. ${ }^{21}$ Treatment with intravenous paclitaxel $135 \mathrm{mg} / \mathrm{m}^{2}$ followed by intravenous cisplatin $75 \mathrm{mg} / \mathrm{m}^{2}$ was compared with intravenous paclitaxel $135 \mathrm{mg} / \mathrm{m}^{2}$ followed by intraperitoneal cisplatin $100 \mathrm{mg} / \mathrm{m}^{2}$, plus intraperitoneal paclitaxel $60 \mathrm{mg} / \mathrm{m}^{2}$ on day 8 . The median survival was 49.7 months in the intravenous arm but 65.6 months in the intraperitoneal arm. However, there was substantially more toxicity in the intraperitoneal arm, including grade 3 and 4 leucopenia, thrombocytopenia, and pain. Only $42 \%$ of patients received all six cycles of planned treatment in the intraperitoneal arm.

Together, these three trials contributed $75 \%$ of the patients in a review of eight trials by the US National Cancer Institute in 2006. This review prompted a clinical announcement stating that intraperitoneal treatment should be considered in optimally debulked patients. However, the approach has not become routine practice. Concerns regarding toxicity and lack of experience in placing and managing indwelling catheters have impeded its acceptance. In addition, it has been suggested that the benefits in GOG 114 are the result of a total of eight cycles of chemotherapy in the intraperitoneal arm, and the benefits in GOG 172 may lie with the additional drug or dose-dense paclitaxel approach in the intraperitoneal arm.

Ongoing trials are evaluating the use of intraperitoneal carboplatin (GOG 252 and iPocc/JGOG 3019) and the use of intraperitoneal therapy with either carboplatin or cisplatin and intraperitoneal paclitaxel after neoadjuvant chemotherapy and interval debulking surgery (NCIC-CTG OV21/ NCRI-PETROC).

\section{Dose-dense chemotherapy}

Increasing the intensity of chemotherapy scheduling has produced some of the most promising results seen in ovarian cancer in the last decade. The rationale for this approach is that, by reducing the interval between treatments, the duration of exposure to the chemotherapeutic agent is increased and there is less time for tumor regrowth. The benefits of this approach were first demonstrated in breast cancer. ${ }^{22,23}$

In 2009, Katsumata et al reported a Phase III study of dose-dense paclitaxel for the Japanese Gynecologic Oncology Group. ${ }^{24}$ The study compared standard 3-weekly carboplatin (AUC 6) and paclitaxel $180 \mathrm{mg} / \mathrm{m}^{2}$ with 3-weekly carboplatin and dose-dense paclitaxel given at $80 \mathrm{mg} / \mathrm{m}^{2}$ every week. The dose-dense arm not only showed an improvement in the primary endpoint of progression-free survival $(28.0$ versus 17.2 months; HR $0.71,95 \%$ confidence interval [CI] $0.58-0.88 ; P=0.0015)$ ) but also in 3-year overall survival (72.1\% versus $65.1 \%$; HR $0.75,95 \%$ CI $0.57-0.98 ; P=0.03$ ). The significant difference in survival was maintained when 
the data were recently updated to a median follow-up of 6.4 years. ${ }^{25}$ The cost of this effect was an increase in toxicity, with greater neutropenia (dose-dense group 92\%, conventional arm $88 \%$ ) and grade 3 or 4 anemia (dose-dense group $69 \%$, conventional arm 44\%) in the dose-dense group. More delays and dose modifications were required and only $61 \%$ received all six cycles as opposed to $73 \%$ of patients in the standard arm. Other side effects, including neurotoxicity, were similar between the two groups.

The effects seen with dose-dense paclitaxel have not been so clearly demonstrated with dose-dense platinum, at least in the first-line setting. Previous studies with weekly cisplatin have not shown an overall survival benefit. ${ }^{26-28}$ A potential explanation is genotypic variation in determinants of drug efficacy, eg, DNA repair gene excision repair cross complementation group 1. Ethnic variations in allele frequencies may impact drug pharmacokinetics and dynamics. ${ }^{29,30}$ The results of confirmatory studies including Caucasian patients are eagerly awaited. The Italian MITO-7 study will evaluate 3-weekly carboplatin (AUC 6) and paclitaxel $175 \mathrm{mg} / \mathrm{m}^{2}$ with weekly carboplatin (AUC 2) plus weekly paclitaxel $80 \mathrm{mg} / \mathrm{m}^{2}$. The National Cancer Research Institute ICON-8 study is a three-arm trial incorporating a conventional treatment arm, and two experimental arms, either 3-weekly carboplatin and weekly paclitaxel or weekly fractionation of both carboplatin and paclitaxel.

A further consideration is that low-dose paclitaxel may exhibit antiangiogenic activity. The ongoing GOG 262 trial allows addition of the antiangiogenic agent, bevacizumab, to two different dosing schedules of paclitaxel in stage II-IV ovarian cancer.

\section{Targeting angiogenesis}

Angiogenesis, the growth of new blood vessels from existing ones, promotes solid tumor growth and metastasis. Epithelial ovarian cancer cells frequently express vascular endothelial growth factor (VEGF), and the level of its expression is an independent prognostic indicator of survival. ${ }^{31}$ Bevacizumab is a humanized monoclonal antibody that inhibits the binding of VEGF-A to its receptors, VEGFR1 and VEGFR2, with resulting inhibition of new vessel formation and regression of tumor vasculature. Tumor responses and delayed progression were seen in single-arm trials. ${ }^{32,33}$ As a result, two front-line Phase III trials were performed and the results were reported in 2011.

In ICON-7, 1528 patients were given carboplatin (AUC 5 or 6 ) and paclitaxel $175 \mathrm{mg} / \mathrm{m}^{2}$ every 3 weeks for six cycles with or without the addition of bevacizumab. ${ }^{34}$
Bevacizumab was given at a dose of $7.5 \mathrm{mg} / \mathrm{kg}$ concurrently every 3 weeks for six cycles and continued for an additional 12 cycles or until disease progression. The majority of patients $(70 \%)$ had stage IIIc or IV ovarian cancer and 30\% were at high risk for progression (stage IIIc or IV with $>1 \mathrm{~cm}$ residual disease following surgery).

In the bevacizumab arm, there was an improvement in median progression-free survival from 17.3 to 19.0 months (HR for progression or death with bevacizumab added, $0.81 ; 95 \%$ CI $0.70-0.94 ; P=0.004$ by the log-rank test). This treatment effect was maximal at 12 months (around the time of completion of bevacizumab) but diminishing by 24 months. The greatest effects were seen in patients at high risk of progression (stage III or IV with $>1 \mathrm{~cm}$ residual disease following surgery), with progression-free survival (restricted mean) at 42 months of 18.1 months with bevacizumab and 14.5 months in the standard arm, and on an interim survival analysis, 36.6 compared with 28.8 months. Final results for overall survival are expected in 2013.

The second trial, GOG 218, had the same standard treatment arm but two experimental arms. ${ }^{35}$ In the bevacizumabthroughout arm, bevacizumab was given with chemotherapy for cycles 2-6 and then continued 3-weekly until a total of 22 cycles. In the bevacizumab-initiation arm, bevacizumab was again given with chemotherapy in cycles 2-6, but placebo was administered in cycles 7-22. The dose of bevacizumab was $15 \mathrm{mg} / \mathrm{kg}$, ie, double the dose in ICON-7. The 1873 women enrolled had either stage III (incompletely resected) or stage IV disease and had undergone debulking surgery.

The median progression-free survival was 10.3 months in the control arm, 11.2 months in the bevacizumab-initiation arm, and 14.1 months in the bevacizumab-throughout group. The HR for progression-free survival comparing the control and bevacizumab-throughout groups was 0.770 (95\% CI 0.681-0.870). However, there were no significant differences in overall survival between the three groups.

The lack of improvement in overall survival in GOG 218 may be due to unblinding of patients at the progression-free survival analysis and crossover to bevacizumab following progression. This did not occur in ICON-7. In GOG 218, the primary endpoint of overall survival was changed to progression-free survival because many investigators and patients contested the blinding of treatment following progression. However, the Fourth Ovarian Cancer Consensus Conference supported the use of progression-free survival as a primary endpoint due to the difficulty of assessing overall 
survival given the confounding effect of subsequent lines of treatment. ${ }^{36}$

Bevacizumab was associated with more adverse events, including hypertension (grade 2 or higher in $18 \%$ of patients in ICON-7, 23\% of patients in GOG 218), an increase in bleeding (mainly mucocutaneous), and thromboembolic events. However, overall, the treatment was well tolerated, and bowel perforations, a concern from earlier bevacizumab studies, were rare ( $1 \%$ of patients in ICON-7, $3 \%$ of patients in GOG 218). ${ }^{37}$

When considering who should receive bevacizumab, the greatest benefit seems to be in those at greatest risk of recurrence, perhaps because the requirement for an additional blood supply is greater in larger tumors than in microscopic deposits after debulking surgery. These results emerged from a preliminary analysis of a subset of the ICON-7 study. Although bevacizumab is licensed for first-line therapy within the European Union, many questions remain about how the drug should best be used. These include the dose and duration of treatment. The use of the lower $7.5 \mathrm{mg} / \mathrm{kg}$ dose in ICON-7 is attractive, but the GOG 218 data suggest that treatment may need to be given for longer. This is supported by the OCEANS study in recurrent, platinum-sensitive ovarian cancer in which bevacizumab was continued until progression $^{38}$ and this has led to a new first-line study comparing 15 months with 30 months of bevacizumab treatment, known as the BOOST (Bevacizumab Ovarian Optimal Standard Treatment) trial. The economic costs of using bevacizumab in the first-line setting have not been thoroughly examined yet. The use of the higher dose of bevacizumab $(15 \mathrm{mg} / \mathrm{kg})$ and increasing the duration of therapy will drive up costs. Economic assessment will also need to evaluate the benefit of extension of progression-free survival, or overall survival if the initial subgroup data from ICON-7 are robust on mature analysis. Further trials will need to explore the integration of bevacizumab into other strategies such as neoadjuvant, dose-dense, and intraperitoneal therapy.

Other first-line trials targeting angiogenesis include the oral VEGF receptor tyrosine kinase inhibitors, pazopanib ${ }^{39}$ or nintedanib. ${ }^{40}$ The trials have completed recruitment and are in follow-up, but the results are not yet available. In another approach, AMG 386, a peptibody that inhibits the interaction of angiopoietin- 1 and angiopoietin-2 with the Tie 2 receptor, is being given with chemotherapy and as maintenance. ${ }^{41}$ Given the potential toxicity, cost, and duration of treatment associated with bevacizumab and other antiangiogenic agents, it is essential that predictive markers for efficacy are identified.

\section{Personalizing therapy}

None of the trials thus far have considered factors predictive of sensitivity to drugs or molecular targeted therapy. Large numbers of patients have been recruited into several frontline trials, and only a small proportion of patients benefit. For example, it is clear that differences in response to standard chemotherapy exist between tumors of varying histology, with advanced mucinous and clear cell cancers having a poorer prognosis than advanced serous carcinomas. ${ }^{42-44}$ A further important distinction is between low-grade and high-grade serous tumors, the latter being more aggressive but more responsive to platinum-based therapy. ${ }^{42}$

To date, large-scale genomic analyses of serous ovarian cancers have not identified high frequency somatic oncogenic driver mutations amenable to targeted intervention. However, it has been suggested that up to $50 \%$ of highgrade serous tumors are deficient in homologous recombination repair of DNA damage as a result of germline (inherited) or somatically acquired $B R C A 1 / 2$ mutations, epigenetic inactivation of $B R C A 1$, or defects in other components of the homologous recombination pathway. An analysis of 489 high-grade serous ovarian adenocarcinomas by the Cancer Genome Atlas Research Network found $20 \%$ to harbor germline or somatic mutations in $B R C A 1 / 2$, and $11 \%$ to lose BRCA1 expression through DNA hypermethylation. A further $25 \%$ showed alterations in other homologous recombination gene, eg, EMSY, PTEN, and RAD51.45

Germline mutations in BRCA1/2 are associated with certain clinical characteristics, known as the BRCAness phenotype. These include young age at presentation, highgrade serous histology, visceral metastases, ${ }^{46}$ platinum sensitivity, ${ }^{47}$ and increased overall survival. ${ }^{48}$ Therefore, it is anticipated that tumors arising from other defects in homologous recombination may exhibit similar characteristics.

Recently poly(adenosine diphosphate-ribose) polymerase (PARP) inhibitors have been shown to provide a mechanism by which homologous recombination deficient cells can be targeted. PARP is an essential component in the repair of single-strand breaks. If inhibited, the persistent single-strand breaks are converted to double-strand breaks which BRCA-deficient cells, deficient in homologous recombination, are unable to repair, resulting in DNA fragmentation and apoptosis. This concept is known as synthetic lethality. ${ }^{49}$ Phase I and II trials are already demonstrating the efficacy of PARP inhibitors, not only in those who carry germline mutations, but also in groups enriched for homologousrecombination deficiency. ${ }^{50}$ 


\section{Conclusion}

Recent results in ovarian cancer trials are encouraging. Neoadjuvant chemotherapy, intraperitoneal delivery, a dosedense approach, and targeting of angiogenesis all present ways in which treatment may be enhanced. Perhaps the more difficult task will be in deciding who will benefit most from which strategy or combination of strategies. In addition to considering stage of disease and histological subtype, the molecular profile of tumors will become increasingly important and the identification of biomarkers to predict efficacy essential.

\section{Disclosure}

The authors report no conflicts of interest in this work.

\section{References}

1. Ferlay J, Shin HR, Bray F, et al. Cancer incidence and mortality worldwide. In: IARC Cancer Base No 10. Lyon, France: International Agency for Research on Cancer; 2010. Available from: http://globocan. iarc.fr. Accessed December 14, 2012.

2. Jemal A, Siegel R, Ward E, Hao Y, Xu J, Thun MJ. Cancer statistics, 2009. CA Cancer J Clin. 2009;59(4):225-249.

3. Goff BA, Mandel LS, Drescher CW, et al. Development of an ovarian cancer symptom index: possibilities for earlier detection. Cancer. 2007;109(2):221-227.

4. Hippisley-Cox J, Coupland C. Identifying women with suspected ovarian cancer in primary care: derivation and validation of algorithm. $B M J$. 2011;344:d8009.

5. Buys SS, Partridge E, Black A, et al. Effect of screening on ovarian cancer mortality: the Prostate, Lung, Colorectal and Ovarian (PLCO) cancer screening randomized controlled trial. JAMA. 2011;305(22):2295-2303.

6. Menon U, Kalsi J, Jacobs I. The UKCTOCS experience - reasons for hope? Int J Gynecol Cancer. 2012;22 Suppl 1:S18-S20.

7. McGuire WP, Hoskins WJ, Brady MF, et al. Cyclophosphamide and cisplatin compared with paclitaxel and cisplatin in patients with stage III and stage IV ovarian cancer. $N$ Engl J Med. 1996;334(1):1-6.

8. Piccart MJ, Bertelsen K, James K, et al. Randomized Intergroup trial of cisplatin-paclitaxel versus cisplatin-cyclophosphamide in women with advanced epithelial ovarian cancer: three-year results. J Natl Cancer Inst. 2000;92(9):699-708.

9. du Bois A, Neijt JP, Thigpen JT. First line chemotherapy with carboplatin plus paclitaxel in advanced ovarian cancer - a new standard of care? Ann Oncol. 1999;10 Suppl 1:35-41.

10. Vasey PA, Jayson GC, Gordon A, et al. Phase III randomized trial of docetaxel-carboplatin versus paclitaxel-carboplatin as first-line chemotherapy for ovarian carcinoma. $J$ Natl Cancer Inst. 2004;96(22):1682-1691.

11. International Collaborative Ovarian Neoplasm Group. Paclitaxel plus carboplatin versus standard chemotherapy with either singleagent carboplatin or cyclophosphamide, doxorubicin, and cisplatin in women with ovarian cancer: the ICON3 randomised trial. Lancet. 2002;360(9332):505-515.

12. Muggia FM, Braly PS, Brady MF, et al. Phase III randomized study of cisplatin versus paclitaxel versus cisplatin and paclitaxel in patients with suboptimal stage III or IV ovarian cancer: a Gynecologic Oncology Group study. J Clin Oncol. 2000;18(1):106-115.

13. du Bois A, Quinn M, Thigpen T, et al. 2004 consensus statements on the management of ovarian cancer: final document of the 3rd International Gynecologic Cancer Intergroup Ovarian Cancer Consensus Conference (GCIG OCCC 2004). Ann Oncol. 2005;16 Suppl 8:viii7-viii12.
14. Bookman MA, Brady MF, McGuire WP, et al. Evaluation of new platinum-based treatment regimens in advanced-stage ovarian cancer: a Phase III trial of the Gynecologic Cancer Intergroup. J Clin Oncol. 2009;27(9):1419-1425.

15. van der Burg ME, van Lent M, Buyse M, et al. The effect of debulking surgery after induction chemotherapy on the prognosis in advanced epithelial ovarian cancer. Gynecological Cancer Cooperative Group of the European Organization for Research and Treatment of Cancer. N Engl J Med. 1995;332(10):629-634.

16. Vergote I, Amant F, Leunen K. Neoadjuvant chemotherapy in advanced ovarian cancer: what kind of evidence is needed to convince US gynaecological oncologists? Gynecol Oncol. 2010;119(1):1-2.

17. Onda T, Matsumoto K, Shibata T, et al. Phase III trial of upfront debulking surgery versus neoadjuvant chemotherapy for stage III/IV ovarian, tubal and peritoneal cancers: Japan Clinical Oncology Group Study JCOG0602. Jpn J Clin Oncol. 2008;38(1):74-77.

18. Vergote I, Trope CG, Amant F, Ehlen T, Reed NS, Casado A. Neoadjuvant chemotherapy is the better treatment option in some patients with stage IIIc to IV ovarian cancer. J Clin Oncol. 2011;29(31): 4076-4078

19. Rauh-Hain JA, Rodriguez N, Growdon WB, et al. Primary debulking surgery versus neoadjuvant chemotherapy in stage IV ovarian cancer. Ann Surg Oncol. 2012;19(3):959-965.

20. Markman M, Bundy BN, Alberts DS, et al. Phase III trial of standarddose intravenous cisplatin plus paclitaxel versus moderately high-dose carboplatin followed by intravenous paclitaxel and intraperitoneal cisplatin in small-volume stage III ovarian carcinoma: an intergroup study of the Gynecologic Oncology Group, Southwestern Oncology Group, and Eastern Cooperative Oncology Group. J Clin Oncol. 2001;19(4): 1001-1007.

21. Armstrong DK, Bundy B, Wenzel L, et al. Intraperitoneal cisplatin and paclitaxel in ovarian cancer. $N$ Engl J Med. 2006;354(1):34-43.

22. Seidman AD, Berry D, Cirrincione C, et al. Randomized phase III trial of weekly compared with every-3-weeks paclitaxel for metastatic breast cancer, with trastuzumab for all HER-2 overexpressors and random assignment to trastuzumab or not in HER-2 nonoverexpressors: final results of Cancer and Leukemia Group B protocol 9840. J Clin Oncol. 2008;26(10):1642-1649.

23. Sparano JA, Wang M, Martino S, et al. Weekly paclitaxel in the adjuvant treatment of breast cancer. $N$ Engl $J$ Med. 2008;358(16): $1663-1671$

24. Katsumata N, Yasuda M, Takahashi F, et al. Dose-dense paclitaxel once a week in combination with carboplatin every 3 weeks for advanced ovarian cancer: a phase 3, open-label, randomised controlled trial. Lancet. 2009;374(9698):1331-1338.

25. Katsumata N, Yasuda M, Isonishi S, et al. Long-term follow-up of a randomized trial comparing conventional paclitaxel and carboplatin with dose-dense weekly paclitaxel and carboplatin in women with advanced epithelial ovarian, fallopian tube, or primary peritoneal cancer: JGOG 3016 trial. J Clin Oncol. 2012;30 Suppl:Abstr 5003.

26. Bolis G, Favalli G, Danese S, et al. Weekly cisplatin given for 2 months versus cisplatin plus cyclophosphamide given for 5 months after cytoreductive surgery for advanced ovarian cancer. J Clin Oncol. 1997;15(5): 1938-1944.

27. Cocconi G, Bella M, Lottici R, et al. Mature results of a prospective randomized trial comparing a three-weekly with an accelerated weekly schedule of cisplatin in advanced ovarian carcinoma. Am J Clin Oncol. 1999;22(6):559-567.

28. Fruscio R, Garbi A, Parma G, et al. Randomized phase III clinical trial evaluating weekly cisplatin for advanced epithelial ovarian cancer. J Natl Cancer Inst. 2011;103(4):347-351.

29. Krivak TC, Darcy KM, Tian C, et al. Single nucleotide polypmorphisms in ERCC1 are associated with disease progression, and survival in patients with advanced stage ovarian and primary peritoneal carcinoma; a Gynecologic Oncology Group study. Gynecol Oncol. 2011;122(1):121-126. 
30. O'Donnell PH, Gamazon E, Zhang W, et al. Population differences in platinum toxicity as a means to identify novel genetic susceptibility variants. Pharmacogenet Genomics. 2010;20(5):327-337.

31. Huang S, Robinson JB, Deguzman A, Bucana CD, Fidler IJ. Blockade of nuclear factor-kappaB signaling inhibits angiogenesis and tumorigenicity of human ovarian cancer cells by suppressing expression of vascular endothelial growth factor and interleukin 8. Cancer Res. 2000;60(19):5334-5339.

32. Burger RA, Sill MW, Monk BJ, Greer BE, Sorosky JI. Phase II trial of bevacizumab in persistent or recurrent epithelial ovarian cancer or primary peritoneal cancer: a Gynecologic Oncology Group Study. J Clin Oncol. 2007;25(33):5165-5171.

33. Cannistra SA, Matulonis UA, Penson RT, et al. Phase II study of bevacizumab in patients with platinum-resistant ovarian cancer or peritoneal serous cancer. J Clin Oncol. 2007;25(33):5180-5186.

34. Perren TJ, Swart AM, Pfisterer J, et al. A phase 3 trial of bevacizumab in ovarian cancer. N Engl J Med. 2011;365(26):2484-2496.

35. Burger RA, Brady MF, Bookman MA, et al. Incorporation of bevacizumab in the primary treatment of ovarian cancer. $N$ Engl J Med. 2011;365(26):2473-2483.

36. Stuart GC, Kitchener H, Bacon M, et al. 2010 Gynecologic Cancer InterGroup (GCIG) consensus statement on clinical trials in ovarian cancer: report from the Fourth Ovarian Cancer Consensus Conference. Int J Gynecol Cancer. 2011;21(4):750-755.

37. Han ES, Monk BJ. What is the risk of bowel perforation associated with bevacizumab therapy in ovarian cancer? Gynecol Oncol. 2007; 105(1):3-6.

38. Aghajanian C, Blank SV, Goff BA, et al. OCEANS: a randomized, double-blind, placebo-controlled phase III trial of chemotherapy with or without bevacizumab in patients with platinum-sensitive recurrent epithelial ovarian, primary peritoneal, or fallopian tube cancer. $J$ Clin Oncol. 2012;30(17):2039-2045.

39. Friedlander M, Hancock KC, Rischin D, et al. A Phase II, open-label study evaluating pazopanib in patients with recurrent ovarian cancer. Gynecol Oncol. 2010;119(1):32-37.
40. Ledermann JA, Hackshaw A, Kaye S, et al. Randomized phase II placebo-controlled trial of maintenance therapy using the oral triple angiokinase inhibitor BIBF 1120 after chemotherapy for relapsed ovarian cancer. J Clin Oncol. 2011;29(28):3798-3804.

41. Karlan BY, Oza AM, Richardson GE, et al. Randomized, double-blind, placebo-controlled phase II study of AMG 386 combined with weekly paclitaxel in patients with recurrent ovarian cancer. J Clin Oncol. 2012; 30(4):362-371.

42. Hess V, A'Hern R, Nasiri N, et al. Mucinous epithelial ovarian cancer: a separate entity requiring specific treatment. J Clin Oncol. 2004;22(6):1040-1044.

43. Lee YY, Kim TJ, Kim MJ, et al. Prognosis of ovarian clear cell carcinoma compared to other histological subtypes: a meta-analysis. Gynecol Oncol. 2011;122(3):541-547.

44. Mackay HJ, Brady MF, Oza AM, et al. Prognostic relevance of uncommon ovarian histology in women with stage III/IV epithelial ovarian cancer. Int J Gynecol Cancer. 2010;20(6):945-952.

45. Cancer Genome Atlas Research Network. Integrated genomic analyses of ovarian carcinoma. Nature. 2011;474(7353):609-615.

46. Gourley C, Michie CO, Roxburgh P, et al. Increased incidence of visceral metastases in scottish patients with BRCA1/2-defective ovarian cancer: an extension of the ovarian BRCAness phenotype. J Clin Oncol. 2010;28(15):2505-2511.

47. Tan DS, Rothermundt C, Thomas K, et al. "BRCAness" syndrome in ovarian cancer: a case-control study describing the clinical features and outcome of patients with epithelial ovarian cancer associated with BRCA1 and BRCA2 mutations. J Clin Oncol. 2008;26(34):5530-5536.

48. Bolton KL, Chenevix-Trench G, Goh C, et al. Association between BRCA1 and BRCA2 mutations and survival in women with invasive epithelial ovarian cancer. JAMA. 2012;307(4):382-390.

49. Banerjee S, Kaye SB, Ashworth A. Making the best of PARP inhibitors in ovarian cancer. Nat Rev Clin Oncol. 2010;7(9):508-519.

50. Ledermann J, Harter P, Gourley C, et al. Olaparib maintenance therapy in platinum-sensitive relapsed ovarian cancer. $N$ Engl J Med. 2012;366(15):1382-1392.
International Journal of Women's Health

\section{Publish your work in this journal}

The International Journal of Women's Health is an international, peerreviewed open-access journal publishing original research, reports, editorials, reviews and commentaries on all aspects of women's healthcare including gynecology, obstetrics, and breast cancer. The manuscript management system is completely online and includes

\section{Dovepress}

a very quick and fair peer-review system, which is all easy to use. Visit http://www.dovepress.com/testimonials.php to read real quotes from published authors. 